Louisiana State University

LSU Digital Commons

$9-4-2013$

\title{
Reconstruction of attosecond electron wave packets using quantum state holography
}

\author{
K. Klünder \\ Lunds Universitet \\ P. Johnsson \\ Lunds Universitet \\ M. Swoboda \\ Technische Universität Dresden \\ A. L'Huillier \\ Lunds Universitet \\ G. Sansone \\ Institute for Photonics and Nanotechnologies, Rome
}

See next page for additional authors

Follow this and additional works at: https://digitalcommons.Isu.edu/physics_astronomy_pubs

\section{Recommended Citation}

Klünder, K., Johnsson, P., Swoboda, M., L'Huillier, A., Sansone, G., Nisoli, M., Vrakking, M., Schafer, K., \& Mauritsson, J. (2013). Reconstruction of attosecond electron wave packets using quantum state holography. Physical Review A - Atomic, Molecular, and Optical Physics, 88 (3) https://doi.org/10.1103/ PhysRevA.88.033404

This Article is brought to you for free and open access by the Department of Physics \& Astronomy at LSU Digital Commons. It has been accepted for inclusion in Faculty Publications by an authorized administrator of LSU Digital Commons. For more information, please contact ir@lsu.edu. 


\section{Authors}

K. Klünder, P. Johnsson, M. Swoboda, A. L'Huillier, G. Sansone, M. Nisoli, M. J.J. Vrakking, K. J. Schafer, and J. Mauritsson

This article is available at LSU Digital Commons: https://digitalcommons.Isu.edu/physics_astronomy_pubs/4881 


\title{
Reconstruction of attosecond electron wave packets using quantum state holography
}

\author{
K. Klünder, ${ }^{1}$ P. Johnsson, ${ }^{1}$ M. Swoboda,${ }^{2}$ A. L'Huillier, ${ }^{1}$ G. Sansone, ${ }^{3}$ M. Nisoli, ${ }^{3}$ M. J. J. Vrakking, ${ }^{4}$ \\ K. J. Schafer, ${ }^{5}$ and J. Mauritsson ${ }^{1, *}$ \\ ${ }^{1}$ Department of Physics, Lund University, P.O. Box 118, SE-221 00 Lund, Sweden \\ ${ }^{2}$ TU Dresden, B CUBE-Center for Molecular Bioengineering, Arnoldstrasse 18, D-01307 Dresden, Germany \\ ${ }^{3}$ Politecnico di Milano, Department of Physics Istituto di Fotonica e Nanotecnologie, CNR-IFN Piazza L. da Vinci 32, 20133 Milano, Italy \\ ${ }^{4}$ Max Born Institute, Max-Born-Straße 2a, D-12489 Berlin, Germany \\ ${ }^{5}$ Department of Physics and Astronomy, Louisiana State University, Baton Rouge, Louisiana 70803-4001, USA
}

(Received 23 June 2013; published 4 September 2013)

\begin{abstract}
We present a method for performing quantum state holography, with which we completely characterize the amplitude and phase of an attosecond electron wave packet. Our approach is an extension of a recent publication [J. Mauritsson et al., Phys. Rev. Lett. 105, 053001 (2010)] in which we demonstrated experimentally that the energies and amplitudes of an attosecond electron wave packet can be characterized using attosecond electron interferometry. Here we show theoretically that attosecond electron interferometry can be extended to retrieve the phases of all the states that make up the wave packet. We demonstrate the feasibility of our method by analyzing a wave packet created by a shake-up process. We show that our method can successfully retrieve arbitrary phases and/or lifetimes added to the component eigenstates.
\end{abstract}

DOI: 10.1103/PhysRevA.88.033404

PACS number(s): 32.80.Rm, 32.80.Qk, 42.65.Ky

\section{INTRODUCTION}

The interaction of ultrafast light pulses with atoms or molecules results in the creation of localized electron wave packets, either through direct excitation or through an indirect process such as shake-up excitation. These electron wave packets can be viewed as a coherent superposition of excited eigenstates, whose initial spatial and temporal localization results from the simultaneous excitation of states spread over a broad bandwidth. The number of states involved, and their initial coherence, determines the subsequent spatial and temporal evolution of the wave packet. If we want to capture and ultimately control localized electron motion, we must therefore be able to characterize broadband wave packets consisting of many excited states spread over many electron volts. A complete reconstruction of such wave packets requires that we characterize both the time-dependent amplitudes and phases of all these states.

Determining the amplitudes and phases of a wavelike object by interference with a reference wave is a well-known approach to wave-packet characterization. It is the essence of holography, and has been used to great advantage in the characterization of optical fields [1]. The method has also been applied to vibrational wave packets in molecules and to highly excited Rydberg wave packets [2-4]. In these systems, the motion takes place on a picosecond to femtosecond time scale. In the latter case, two bound wave packets were used, one the object to be measured and the other a known reference that spectrally overlapped the unknown one. One challenge in attosecond science is to develop similar techniques, which in addition do not require the reference wave packet to interfere with the unknown wave packet while it is still bound. This is a challenge because we need to produce a known reference, like the plane wave used in regular holography, or a Gaussian excitation pulse with no additional phases

*johan.mauritsson@fysik.lth.se used in quantum state holography. We propose a method to solve this problem by having the reference be a free wave packet, which does not interfere with the unknown wave packet during the evolution of the electron dynamics of interest, but only in the course of the detection step, when the bound wave packet is ionized by a coherent probe pulse and the resulting angle- and energy-resolved photoelectron spectrum is measured.

In a recent publication [5], we experimentally demonstrated an interferometric pump-probe technique that we called attosecond electron interferometry. Similar interferometric approaches have subsequently been presented by Choi and co-workers [6] and by Xie and co-workers [7]. We showed that we could spectrally resolve and determine the population of a number of states of a bound wave packet (the $n p$ series in helium starting from the $3 p$ state). In the experiment, we could not retrieve the phases of the individual states due to an insufficient signal-to-noise ratio, but the interferometric nature of the method implies that this phase information should in fact be embedded in the recorded interferogram. Here, we demonstrate theoretically that the technique can indeed be extended to quantum state holography where both the amplitudes and the phases of the states in the excited electron wave packet are determined.

Our phase retrieval method is an extension of the recent publication by Kim and co-workers [8] where interferometry was used to analyze the amplitude and phase of a single excited state. Functional holography of a wave packet requires that we characterize all the states in the superposition. In this article, we demonstrate that our holographic technique works irrespective of the way the wave packet is created. We show this by using a simplified model of a shake-upshake-off process, where we successfully retrieve both the time-dependent amplitudes and the phases of all the bound states in the coherent superposition.

The basic idea of attosecond electron interferometry is illustrated in Fig. 1. When an attosecond pulse interacts 


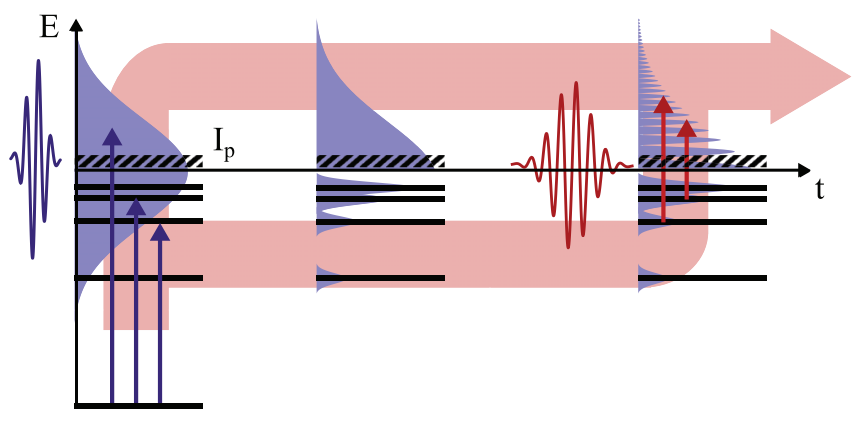

FIG. 1. (Color online) Principle of the attosecond electron interferometer. A bound wave packet is created either directly by excitation with an attosecond pulse or through an intra-atomic or intra-molecular process such as shake-up resulting from ionization of the system by the attosecond pulse. Simultaneously, a continuum reference wave packet is created. Both wave packets evolve freely in time until, after a variable delay, the bound wave packet is ionized using a synchronized probe pulse. At this point, quantum-mechanical interference arises between the two (direct and indirect) pathways that produce the continuum electrons.

with an atom or a molecule at time $t_{0}$, it excites a broad coherent superposition of bound states spread over several electron volts. This can either happen by direct excitation or as a subsidiary process, e.g., by shake-up [9] following the ionization of a core electron. The coherent superposition, and hence the electron dynamics that unfold, is completely characterized by the populations of these states (as given by the square modulus of the amplitude) and their relative phases, i.e.,

$$
\Psi_{b}\left(t>t_{0}\right)=\sum_{i} A_{i} \Psi_{i} e^{-i\left[E_{i}\left(t-t_{0}\right) / \hbar-\varphi_{i}\right]},
$$

where $E_{i}$ and $\Psi_{i}$ are the bound state energies and wave functions, and $\varphi_{i}$ and $A_{i}$ are the phases and amplitudes.

Because of the very broad spectral width of the attosecond extreme ultraviolet (xuv) light pulses, the creation of a bound state wave packet is almost always accompanied by the creation of a continuum wave packet. This continuum wave packet will serve as the reference wave packet in our method. After the initial excitation, both wave packets evolve freely in time. The bound wave packet is probed through a further excitation step after a variable delay $t$ using a probe pulse that is locked in phase with the attosecond laser pulse that initially created the wave packet. The subsequent ionization leads to interference between the continuum wave packet that is directly created by the attosecond pulse and the continuum wave packet that is indirectly created as a result of ionization by the probe laser pulse of the bound wave packet. In this article, we demonstrate that it is possible to determine the amplitudes $A_{i}$ including possible lifetime effects of the bound states, $\Psi_{i}$, the energies, $E_{i}$, and the initial phases, $\varphi_{i}$.

This article is organized as follows. In Sec. II the simplified shake-up model is described together with the pulse parameters used in the calculations, followed by a brief explanation of how we analyze the resulting interferograms. In Sec. III the separation of the angular resolved data into Legendre polynomials is presented, including a method to separate different ionization pathways. This is followed in Sec. IV by an explanation of how the encoded lifetime and phase information can be retrieved from the spectrograms, ending with conclusions in Sec. V.

\section{ANALYSIS OF A SHAKE-UP WAVE PACKET}

Shake-up processes can occur after the sudden removal of one electron, e.g., by photoionization with high-energy photons [9]. The abrupt changes in the atomic potential create a perturbation that may "shake up" the remaining electron(s) into an excited bound or even continuum state ("shake-off"). We simulated such a shake-up and shake-off process in helium using a sudden approximation, consisting of an instantaneous removal of one electron and projecting the wave function of the other electron onto the eigenstates of the ionic system [10]. This process produces a wave packet that is a coherent superposition of $n s$ bound states and a continuum $s$ wave packet [see Fig. 2(a)]. We will assume that direct photoionization of the atom does not produce any photoelectrons with kinetic energies overlapping the kinetic energies of the shake-off electrons. As illustrated in Fig. 2(b), we further ionize the excited bound states after a variable delay by a two-cycle pulse with a $200 \mathrm{~nm}$ central wavelength and $a \cos ^{2}$ electric field envelope, which corresponds to a bandwidth of $6 \mathrm{eV}$ full width at half-maximum (FWHM) and a duration of just below half a femtosecond. The central wavelength is chosen in order to ionize most of the excited states in the helium ion with only one photon. Recent advances in source development indicate that ultrashort pulses in this wavelength regime are now experimentally feasible [11]. We calculated the angularly resolved electron spectra $\mathcal{F}(E, \theta, t)$ as a function of delay, $t$, between the shake-up-shake-off excitation (pump) and the probe laser pulse by solving the time-dependent Schrödinger equation $[12,13]$. $E$ denotes the final energy, while $\theta$ is the angle between the direction of emission of the electron and the direction of the common polarization of the xuv pump and uv probe laser fields, which coincides with the quantization axis. Note that due to the cylindrical symmetry of the problem, $\mathcal{F}(E, \theta, t)$ does not depend on the azimuthal angle $\phi$. The methods employed to solve the time-dependent Schrödinger equation (TDSE) and

(a)
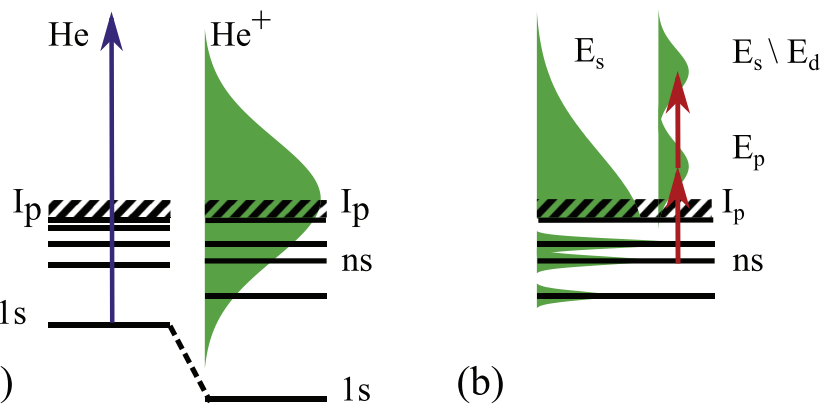

FIG. 2. (Color online) Electron interferometry for the shake-up excitation in helium. (a) The rapid removal of an electron excites the remaining electron to excited bound and continuum states of the ion. (b) Ionization of the bound states with one or two probe photons after the shake-up excitation. 

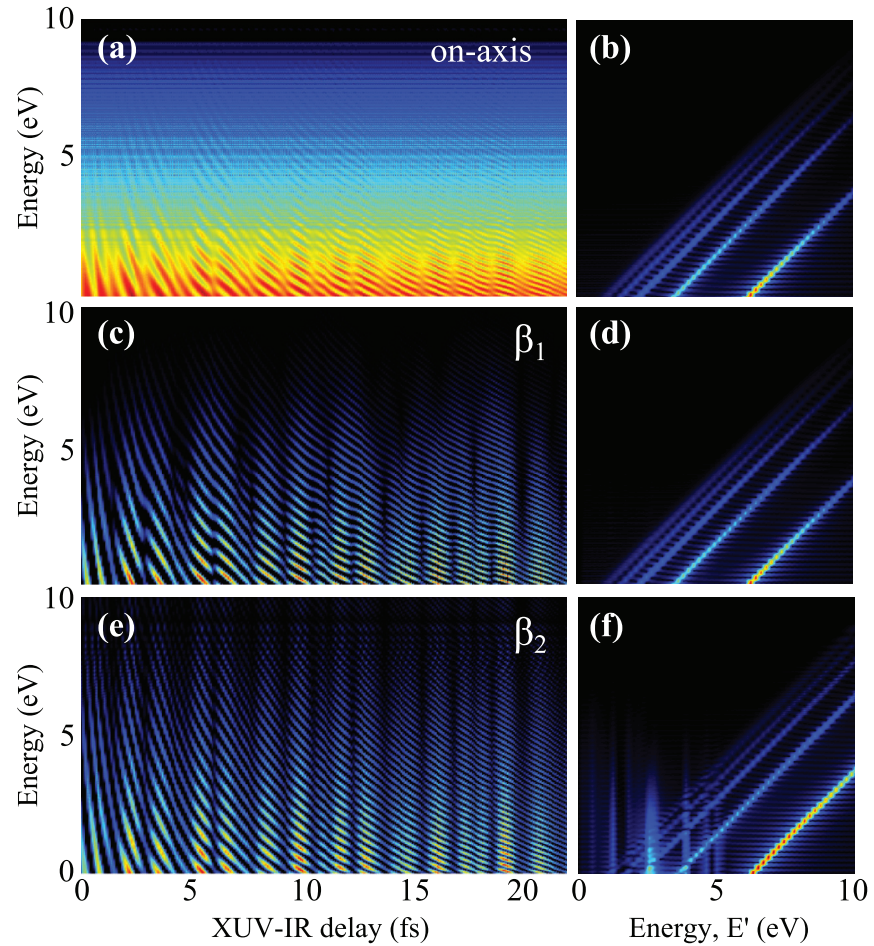

FIG. 3. (Color online) Analysis of the two-color photoelectron interferograms that are calculated for a shake-up wave packet in a helium ion. (a) Complete on-axis spectrogram. (b) Fourier analysis showing the components of the wave packet. (c) $\beta_{1}(E, t)$ and the corresponding Fourier analysis (d). (e) $\beta_{2}(E, t)$ and (f) its Fourier analysis. Different interference phenomena exhibit distinct angular distributions. While interferences between processes of opposite parity are described by $\beta_{1}(E, t)$, those between processes of the same parity appear in even expansion coefficients, e.g., quantum beats as vertical lines in (f).

how to extract the angularly resolved electron spectra from the time-dependent wave function is described in detail in [14].

In Fig. 3(a), the results of the calculations are presented for electrons emitted in the direction of the polarization vector. The electron signal in this direction is plotted as a function of $E$ and $t$. The spectrogram $\mathcal{F}(E, \theta, t)$ (here with $\theta=0$ ) exhibits a series of tilted interference fringes, the tilt varying from almost vertical at small time delays to almost horizontal at large delays. We analyze the interferences by performing a Fourier transformation along the delay axis for all final energies [5]. The result shown in Fig. 3(b) is a two-dimensional function $S\left(E, E^{\prime}\right)$ of the continuum energy $E$ and of energy $E^{\prime}$, the conjugate variable of $t$ in the Fourier transform. This function exhibits a series of tilted lines at $45^{\circ}$ corresponding to interferences between the wave packet ionized by the probe pulse and the reference continuum wave packet created by the shake-off process. Note that due to the opposite parity of the two pathways, these direct-indirect interferences are not present in an angle-integrated measurement.

The structure of the interference pattern both in the $\{E, t\}$ interferogram and in the $\left\{E, E^{\prime}\right\}$ representation can be understood by considering the accumulated phase difference $\Delta \Phi$ along the two branches of the interferometer (see Fig. 1). Considering a final energy $E$ and an excited bound state $E_{i}$, the accumulated phase difference at time $t$ is

$$
\Delta \Phi(E, t)=\left(E-E_{i}\right)\left(t-t_{0}\right) / \hbar+\varphi_{i}+\delta \phi(E),
$$

where we included for the sake of completeness the phase $\delta \phi(E)=\phi_{\text {dir }}(E)-\phi_{\text {ind }}(E)$ as a possible phase difference that results from the ionization processes that produce the direct and the indirect continuum wave packets. The phases $\phi_{\text {dir }}(E)$ and $\phi_{\text {ind }}(E)$ are associated, respectively, with the shake-off ionization and the delayed photoionization with the probe pulse [15-17]; compare Fig. 2. The phases, $\varphi_{i}$, which characterize the bound wave packet are those that we want to determine using our interferometric technique. Note that we are only interested in the relative phases of the wave-packet components. Let us therefore, in a first step and for the sake of simplicity, neglect any state and energy dependence of $\delta \phi(E)$ over the range considered. We will also assume that the probe pulses are transform-limited so that $\delta \phi$ can be treated as a small constant phase offset, which does not alter the relative phase difference of the wave-packet components.

The position of the interference fringes is determined by the families of hyperbolic curves $E=E_{i}+\left(n \pi-\varphi_{i}\right) \hbar /\left(t-t_{0}\right)$, with $n$ an integer. For a given energy, $E$, the oscillation frequency, given by $\left(E-E_{i}\right) / \hbar$, depends linearly on the photoelectron kinetic energy $E$, leading to straight lines with a tilt of $45^{\circ}$ in the Fourier plane, which intersects for $E=0$ at the absolute value of the bound state energies, $\left|E_{i}\right|$. In this way, we can identify the $3 s, 4 s, 5 s, 6 s$, and $7 s$ states with the binding energies $6.04,3.40,2.18,1.50$, and $1.10 \mathrm{eV}$ with an accuracy given by the numerical resolution. The relative strength of each Fourier component is directly related to its relative contribution to the wave packet. Note that no information is obtained for the population of the $2 s$ state since the photon energy of the probe pulse is not sufficient to ionize it.

\section{LEGENDRE ANALYSIS}

A deeper understanding of the interference pattern can be achieved by performing a Legendre analysis of the calculated angular distributions. To the extent that they are produced by the absorption of a single xuv photon and a single uv photon, and since here we only need to consider the magnetic quantum number, $m=0, \mathcal{F}(E, \theta, t)$ can be numerically decomposed as

$$
\mathcal{F}(E, \theta, t)=\sum_{\ell=0}^{2} \beta_{\ell}(E, t) P_{\ell}^{0}(\cos \theta),
$$

where the functions $P_{\ell}^{0}$ are Legendre polynomials, which are related to the spherical harmonics by

$$
P_{\ell}^{0}(\cos \theta)=\sqrt{\frac{4 \pi}{2 \ell+1}} Y_{\ell}^{0}(\theta)
$$

Figures 3(c) and 3(e) show the extracted coefficients $\beta_{1}(E, t)$ and $\beta_{2}(E, t)$ together with the Fourier analysis carried out for these coefficients, leading, respectively, to $S_{1}\left(E, E^{\prime}\right)$ and $S_{2}\left(E, E^{\prime}\right)$, which are shown in Figs. 3(d) and 3(f). The hyperbolic fringes appear clearly in both $\beta_{1}(E, t)$ and $\beta_{2}(E, t)$. In Figs. 3(c) and 3(e), they are modulated in intensity due to the presence of several bound states. The Fourier transforms $S\left(E, E^{\prime}\right)$ show lines at $45^{\circ}$ which are characteristic of directindirect interferences as well as a few vertical lines in Fig. 3(f) 
which can be identified as quantum beats between the bound state components [5]. The quantum beat signals are due to beating between outgoing electron waves from different bound states and therefore do not depend on the detection energy. It is clear from Figs. 3(d) and 3(f) that the quantum beats only appear in the even Legendre polynomials. Figures 3(c) and 3(e), however, look very similar, but this is a superficial effect similar to Moiré patterns.

To understand the information contained in this analysis, we express the shake-off amplitude for the ejection of an electron in the direction given by $(\theta)$ as

$$
M_{\mathrm{dir}}(\theta)=\alpha_{E} Y_{0}^{0}(\theta) e^{-i E\left(t-t_{0}\right) / \hbar} .
$$

Here we do not include electrons that are directly ionized by the xuv pulse, since these have a much higher final energy. Similarly, the amplitude for shake-up into states $i$ or $j$ (we only consider two states here) followed by ionization by the probe field can be written as

$$
M_{\text {ind }}(\theta)=\left[\alpha_{i} e^{-i E_{i}\left(t-t_{0}\right) / \hbar+i \varphi_{i}}+\alpha_{j} e^{-i E_{j}\left(t-t_{0}\right) / \hbar+i \varphi_{j}}\right] Y_{1}^{0}(\theta) .
$$

In Eqs. (5) and (6), $\alpha_{E}, \alpha_{i}$, and $\alpha_{j}$ are the probability amplitudes for the different processes. In general, these are complex quantities that include ionization phases discussed above. The interference signal is proportional to $\left|M_{\text {dir }}(\theta)+M_{\text {ind }}(\theta)\right|^{2}$. We identify the coefficients of the Legendre polynomial expansion for this simple case as

$$
\begin{aligned}
\beta_{0}(E, t) \propto & \left|\alpha_{E}\right|^{2}+\left|\alpha_{i}\right|^{2}+\left|\alpha_{j}\right|^{2} \\
& +2 \operatorname{Re}\left\{\alpha_{i} \alpha_{j}^{*} e^{i\left(E_{j}-E_{i}\right)\left(t-t_{0}\right) / \hbar+i\left(\varphi_{i}-\varphi_{j}\right)}\right\}, \\
\beta_{1}(E, t) \propto & \operatorname{Re}\left\{\alpha_{E}^{*} \alpha_{i} e^{i\left(E-E_{i}\right)\left(t-t_{0}\right) / \hbar+i \varphi_{i}}\right. \\
& \left.+\alpha_{E}^{*} \alpha_{j} e^{i\left(E-E_{j}\right)\left(t-t_{0}\right) / \hbar+i \varphi_{j}}\right\}, \\
\beta_{2}(E, t) \propto & \left|\alpha_{i}\right|^{2}+\left|\alpha_{j}\right|^{2} \\
& +2 \operatorname{Re}\left\{\alpha_{i} \alpha_{j}^{*} e^{i\left(E_{j}-E_{i}\right)\left(t-t_{0}\right) / \hbar+i\left(\varphi_{i}-\varphi_{j}\right)}\right\} .
\end{aligned}
$$

Processes that have different parity, such as the interference between shake-off and ionization of the shake-up wave packet by one-photon absorption, appear in the odd coefficient $\beta_{1}(E, t)$. Quantum beats between states $i$ and $j$ show up in the even coefficients $\beta_{0}(E, t)$ and $\beta_{2}(E, t)$. Additionally, $\beta_{0}(E, t)$ (not plotted) has a strong background contribution $\left|\alpha_{E}\right|^{2}$ from the shake-off, which is not present in $\beta_{2}(E, t)$.

In addition to the quantum beats that are clearly visible in the Fourier analysis in Fig. 3(f), we also see lines at $45^{\circ}$ in this channel that are typical for the direct-indirect interferences with the reference wave packet. The $45^{\circ}$ lines appear at a higher energy compared to Fig. 3(d) since these are due to ionization of the shake-up wave packet by the absorption of two-probe photons. Figure 2(b) indicates the different processes that must be considered. The probability amplitude corresponding to two-photon absorption with final angular momentum $\ell(\ell=$ $0,2)$ can be expressed as

$M_{\mathrm{ind}}^{(2)}(\theta)=\left[\alpha_{i, \ell}^{(2)} e^{-i E_{i}\left(t-t_{0}\right) / \hbar+i \varphi_{i}}+\alpha_{j, \ell}^{(2)} e^{-i E_{j}\left(t-t_{0}\right) / \hbar+i \varphi_{j}}\right] Y_{\ell}^{0}(\theta)$,

where $\alpha_{i, \ell}^{(2)}$ indicates the probability amplitude for shake-up to the $i$ state, followed by the above-threshold two-photon ionization including $\phi_{\text {ind }}^{(2)}$ as the associated two-photon ionization phase $[16,18]$, which, for simplicity, we assume to be stateand energy-independent. When considering two-probe-photon ionization, $\beta_{1}(E, t)$ is unchanged while $\beta_{2}(E, t)$ becomes

$$
\begin{aligned}
\beta_{2}^{(2)}(E, t) \propto & \left|\alpha_{i}\right|^{2}+\left|\alpha_{j}\right|^{2}+2 \operatorname{Re}\left\{\alpha_{i} \alpha_{j}^{*} e^{i\left(E_{j}-E_{i}\right)\left(t-t_{0}\right) / \hbar+i\left(\varphi_{i}-\varphi_{j}\right)}\right\} \\
& +2 \operatorname{Re}\left\{\alpha_{E}^{*} \alpha_{i, 2}^{(2)} e^{i\left(E-E_{i}\right)\left(t-t_{0}\right) / \hbar+i \varphi_{i}}\right. \\
& \left.+\alpha_{E}^{*} \alpha_{j, 2}^{(2)} e^{i\left(E-E_{j}\right)\left(t-t_{0}\right) / \hbar+i \varphi_{j}}\right\}
\end{aligned}
$$

We see from this that $\beta_{2}(E, t)$ includes both quantum beats and direct-indirect interferences involving two-probe-photon absorption. Note that both the quantum beats and the interferences involving absorption of two-probe photons scale with the square of the amplitude of the probe field $\left|E_{\text {probe }}\right|^{2}$.

\section{LIFETIME AND PHASE RETRIEVAL}

To illustrate our technique, and that it can be used to resolve bound state dynamics, we artificially impose a very short lifetime of $5 \mathrm{fs}$ on the $3 \mathrm{~s}$ state and check that we can retrieve this lifetime using our method. We give the $3 s$ state a lifetime by applying a non-Hermitian operator to the wave function at every time step. Like a mask function that removes probability from the total wave function, this operator removes amplitude from only the $3 \mathrm{~s}$ part of the total wave function. The removal is done in the following way. At each time step, $t_{n}=n \Delta t$, the wave function $\left|\psi_{n}\right\rangle$ is propagated forward in time by applying the short-time propagator $U\left(t_{n+1 / 2} ; \Delta t\right)$, which solves the TDSE using the Hamiltonian at time $t_{n+1 / 2}=(n+1 / 2) \Delta t$. We insert a state-specific mask function into the time evolution, which then reads

$$
\left|\psi_{n+1}\right\rangle=\left(1-\frac{\Delta t}{2 \tau}|3 s\rangle\langle 3 s|\right) U\left(t_{n+1 / 2} ; \Delta t\right)\left|\psi_{n}\right\rangle .
$$

The parameter $\tau$ is the lifetime of the $3 s$ state, in the sense that repeated application of the algorithm will lead, in the limit of small $\Delta t$, to an exponential decrease in the probability of finding the electron in the $3 s$ state as $e^{-t / \tau}$, where $t$ is the propagation time.

To access the time dependence of the $3 s$ state using our retrieval method, the $3 s$ contribution to the expansion coefficient $\beta_{1}(E, t)$ is filtered out by applying a window function to $S_{1}\left(E, E^{\prime}\right)$ and then an inverse Fourier transform back to the time domain. Figure 4 illustrates the different steps. In Fig. 4(a), $\beta_{1}(E, t)$ is shown with the corresponding Fourier function $S_{1}\left(E, E^{\prime}\right)$ in Fig. 4(b). The edges of the filter function are indicated as dashed lines in Fig. 4(b). $S_{1}\left(E, E^{\prime}\right)$, after filtering, is inverse Fourier transformed back to the time domain. The result is shown in Fig. 4(c). The separated $3 \mathrm{~s}$ signal shows a clear decay. From the integrated signal, we determine a lifetime of $5 \mathrm{fs}$, in agreement with the imposed one.

Finally, we explain how to retrieve the phases of the wavepacket components from this type of measurement. We again make use of the fact that we can separate out all of the states via the Fourier analysis, which yields $S_{1}\left(E, E^{\prime}\right)$. Window functions are then used to filter out the contributions from each single state as explained in Figs. 4(a)-4(c). For a given final energy, $E$, the phase of the oscillation is given by Eq. (2). The additional phase term due to the ionization can be neglected because we have assumed it to be state- and energy-independent [see the discussion following Eq. (2)]. We can do this because 

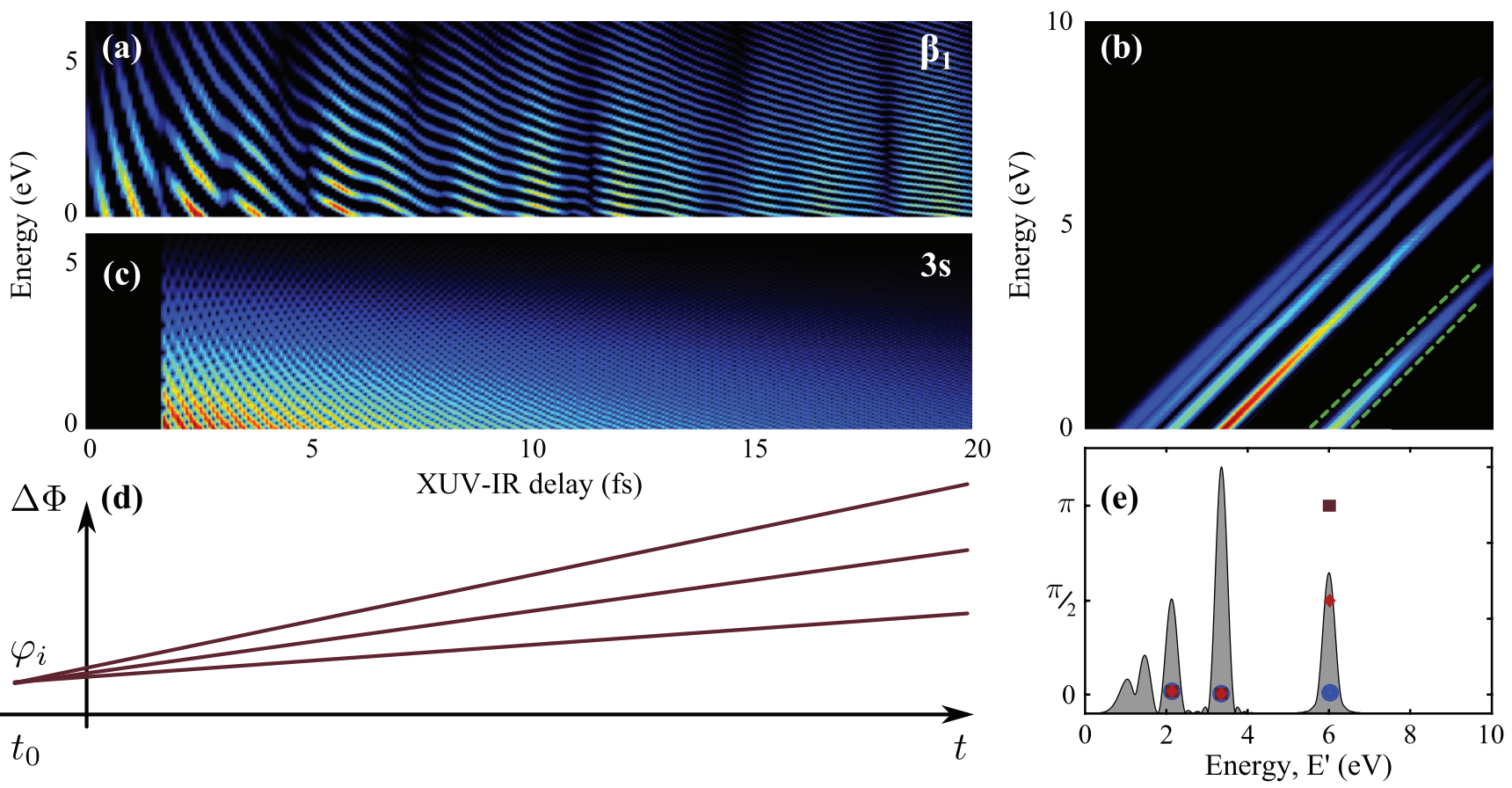

FIG. 4. (Color online) Lifetime analysis and phase retrieval. (a) $\beta_{1}(E, t)$ for shake-up excitation in a helium ion. (b) Fourier analysis of $\beta_{1}(E, t)$ and an example of a window function (green, dashed lines) to separate single states. (c) Back transformation into the time domain for the separated $3 s$ state with a lifetime of $5 \mathrm{fs}$. (d) Idea of the phase retrieval: $\Delta \Phi$ for different continuum energies. The evolution of $\Delta \Phi$ depends on the initial phase at time $t_{0}$. (e) Spectrum at threshold and retrieved phases for three calculations under different initial conditions.

we compare the interference signal for all the states at the same final energy. This means that the additional phase leads to a constant offset that is the same for all the states (see also the previous discussion). An illustration of the phase evolution is sketched in Fig. 4(d). The measured phase $\Delta \Phi(E, t)$ for one state $\varphi_{i}$ in the wave packet is plotted for three different observation energies $E$. The slope of each line is given by the difference between continuum and binding energy, $E-E_{i}$. The phase evolution is linear and can be traced back to the initial excitation time $t_{0}$ where all lines will intersect. The phase offset at $t_{0}$ is then the initial phase $\varphi_{i}$.

To correctly retrieve the phase, $\varphi_{i}$, we therefore need to know precisely the energy of the state, $E_{i}$, and the exact time of creation of the reference wave packet, $t_{0}$. This information can be obtained by utilizing the fact that we measure $\Delta \Phi(E, t)$ as a function of both delay and energy. $E_{i}$ is already extracted from the Fourier transform, while $t_{0}$ is obtained by differentiating $\Delta \Phi(E, t)$ with respect to $E$, yielding $t-t_{0}$, from which $t_{0}$ can be determined [see Eq. (2)]. We differentiate $\Delta \Phi(E, t)$ for observation energies from threshold up to $1 \mathrm{eV}$. In our calculations, the sudden shake-up takes place at $-0.66 \mathrm{fs}$, or one period of the probe field, and the value we find for $t_{0}$ by differentiating $\Delta \Phi(E, t)$ agrees very well with this. We imposed an initial phase of $0, \pi / 2$, and $\pi$ to the $3 s$ state for different runs of our calculation while leaving all other parameters unchanged. The result of our phase retrieval is shown in Fig. 4(e). The figure presents the three reconstructed wave packets. As expected, the initial phases for the $4 s$ and $5 s$ state are unaffected for all wave packets, while the initial phases for the $3 \mathrm{~s}$ state can be retrieved within numerical precision to $0.01 \pi, 0.497 \pi$, and $0.996 \pi$.
The phase retrieval method presented here relies on the fact that we measure the phase difference between the reference wave packet and the bound wave packet via a delayed ionization. As a consequence, it is also possible to compare the phase difference with respect to other pathways, namely the two-photon ionization shown in Fig. 2(b). In our calculations, the one- and two-photon ionization paths overlap substantially in energy due to the very short probe pulses used, but the signals can still be separated since they appear in different Legendre coefficients. While the $\beta_{1}(E, t)$ coefficient contains information about the one-photon case, the $\beta_{2}(E, t)$ coefficients give us access to the two-photon contribution [compare Eqs. (7) and (9)]. Assuming an identical phase imprint for the one-photon ionization, the phase difference between the one- and two-photon ionization $\left(\phi_{\text {ind }}-\phi_{\text {ind }}^{(2)}\right)$ would yield solely the phase contribution due to the second photon. This would mean that we have a direct method to measure the phase related to the measurement process in time-delay measurements [16], which could be of great interest.

\section{CONCLUSION}

In conclusion, we have demonstrated that attosecond electron interferometry can be extended to quantum state holography and that the wave function of a quantum system can be retrieved in both amplitude and phase. Here we have illustrated the technique using a sudden shake-up model, but it can work equally well for different types of excitations. An experimental realization of this method is challenging but well within reach. The technique presented is also useful when analyzing the recent data with transient absorption where similar features appear $[19,20]$. 


\section{ACKNOWLEDGMENTS}

This research was supported by the Swedish Foundation for Strategic Research, the Marie Curie program ATTOFEL (ITN), the European Research Council (ALMA), the Swedish
Research Council, the National Science Foundation through Grant No. PHY-0701372, and the Knut and Alice Wallenberg Foundation. K.J.S. acknowledges support from the Ball Family Professorship.
[1] M. Sandtke and L. Kuipers, Nat. Photon. 1, 573 (2007).

[2] C. Leichtle, W. P. Schleich, I. Sh. Averbukh, and M. Shapiro, Phys. Rev. Lett. 80, 1418 (1998).

[3] T. C. Weinacht, J. Ahn, and P. H. Bucksbaum, Phys. Rev. Lett. 80, 5508 (1998).

[4] T. C. Weinacht, J. Ahn, and P. H. Bucksbaum, Nature (London) 397, 233 (1999).

[5] J. Mauritsson, T. Remetter, M. Swoboda, K. Klunder, A. L'Huillier, K. J. Schafer, O. Ghafur, F. Kelkensberg, W. Siu, P. Johnsson, M. J. J. Vrakking, I. Znakovskaya, T. Uphues, S. Zherebtsov, M. F. Kling, F. Lepine, E. Benedetti, F. Ferrari, G. Sansone, and M. Nisoli, Phys. Rev. Lett. 105, 053001 (2010).

[6] N. N. Choi, T. F. Jiang, T. Morishita, M.-H. Lee, and C. D. Lin, Phys. Rev. A 82, 013409 (2010).

[7] X. Xie, S. Roither, D. Kartashov, E. Persson, D. G. Arbo, L. Zhang, S. Grafe, M. S. Schoffler, J. Burgdorfer, A. Baltuska, and M. Kitzler, Phys. Rev. Lett. 108, 193004 (2012).

[8] K. T. Kim, D. H. Ko, J. Park, N. N. Choi, C. M. Kim, K. L. Ishikawa, J. Lee, and C. H. Nam, Phys. Rev. Lett. 108, 093001 (2012).

[9] V. Schmidt, Rep. Prog. Phys. 55, 1483 (1992).

[10] T. Schneider, P. L. Chocian, and J.-M. Rost, Phys. Rev. Lett. 89, 073002 (2002).

[11] A. Wirth et al., Science 334, 195 (2011).
[12] K. J. Schafer and K. C. Kulander, Phys. Rev. Lett. 78, 638 (1997).

[13] K. Kulander and T. Rescigno, Comput. Phys. Commun. 63, 523 (1991).

[14] K. J. Schafer, in Strong Field Laser Physics, edited by T. Brabec (Springer Science + Media Business, New York, 2008).

[15] D. J. Kennedy and S. T. Manson, Phys. Rev. A 5, 227 (1972).

[16] K. Klünder, J. M. Dahlström, M. Gisselbrecht, T. Fordell, M. Swoboda, D. Guénot, P. Johnsson, J. Caillat, J. Mauritsson, A. Maquet, R. Taïeb, and A. L'Huillier, Phys. Rev. Lett. 106, 143002 (2011).

[17] D. Guénot, K. Klünder, C. L. Arnold, D. Kroon, J. M. Dahlström, M. Miranda, T. Fordell, M. Gisselbrecht, P. Johnsson, J. Mauritsson, E. Lindroth, A. Maquet, R. Taïeb, A. L'Huillier, and A. S. Kheifets, Phys. Rev. A 85, 053424 (2012).

[18] J. M. Dahlström, D. Guénot, K. Klünder, M. Gisselbrecht, J. Mauritsson, A. L'Huillier, A. Maquet, and R. Taïeb, Chem. Phys. 414, 53 (2013).

[19] S. Chen, M. J. Bell, A. R. Beck, H. Mashiko, M. Wu, A. N. Pfeiffer, M. B. Gaarde, D. M. Neumark, S. R. Leone, and K. J. Schafer, Phys. Rev. A 86, 063408 (2012).

[20] M. Chini, X. Wang, Y. Cheng, Y. Wu, D. Zhao, D. A. Telnov, S. Chu, and Z. Chang, Sci. Rep. 3, 1105 (2013). 